\title{
Monitoreo de la Corrosión de Aceros Embebidos en Concretos obtenidos a partir de Subproductos Industriales
}

\author{
Willian Aperador $^{(1)}$, Jorge H. Bautista-Ruiz ${ }^{(2)}$, Arnoldo Emilio Delgado ${ }^{(1,3)}$ \\ (1) Departamento de Ingeniería, Universidad Militar Nueva Granada, Carrera 11 No. 101-80, Bogotá- \\ Colombia. (e-mail:g.ing.materiales@gmail.com) \\ (2) Departamento de Física, Universidad Francisco de Paula Santander. Avenida Gran Colombia No 12E - \\ 96. B Colsag. Edificio de Laboratorios, San José de Cúcuta-Colombia \\ (e-mail: jorgebautista@ufps.edu.co) \\ (3) Facultad de Ingeniería Industrial, Escuela Colombiana de Ingeniería“Julio Garavito", AK.45 No.205-59 \\ (Autopista Norte), Bogotá-Colombia( e-mail: arnoldo.delgado@unimilitar.edu.co)
}

Recibido Feb. 13, 2013; Aceptado Abr. 16, 2013; Versión final recibida Abr. 25, 2013

\begin{abstract}
Resumen
Se evaluó la corrosión del acero estructural ASTM A 706 embebido en concretos obtenidos de subproductos industriales como escorias siderúrgicas y cenizas volantes activadas alcalinamente. En el estudio se emplearon técnicas de caracterización como potencial de circuito abierto, resistencia a la polarización lineal y espectroscopía de impedancia electroquímica en una disolución de cloruro de sodio al 3,5\%. La interface acero-concreto fue caracterizada por espectroscopía Mössbauer de transmisión a temperatura ambiente y difracción de rayos $X$. Los resultados obtenidos por las diferentes técnicas de caracterización se compararon con valores obtenidos para concretos de cemento Portland. Los productos de corrosión identificados corresponden a magnetita $\left(\mathrm{Fe}_{3} \mathrm{O}_{4}\right)$, wuestita $(\mathrm{FeO})$ y goethita $(\alpha-\mathrm{FeOOH})$.
\end{abstract}

Palabras clave:cenizas volantes, escoria siderúrgica, espectroscopía de impedancia electroquímica, difracción de rayos $X$, espectroscopía Mössbauer

\section{Monitoring Corrosion of Concrete Embedded Steel obtained from Industrial Product Wastes}

\begin{abstract}
Corrosion of ASTM A 706 structural steel embedded in concrete obtained from by-products as steel slag and fly ash alkali activated was evaluated. Characterization techniques such as open circuit potential, linear polarization resistance and electrochemical impedance spectroscopy in a solution of sodium chloride $3.5 \%$ were employed. The steel-concrete interface was characterized by transmission Mössbauer spectroscopy at room temperature and X-ray diffraction. The results obtained by the different characterization techniques were compared with values obtained for Portland cement concrete. The corrosion products identified are magnetite $\left(\mathrm{Fe}_{3} \mathrm{O}_{4}\right)$, wuestite $(\mathrm{FeO})$ and goethite $(\alpha-\mathrm{FeOOH})$.
\end{abstract}

Keywords: fly ash, slag, electrochemical impedance spectroscopy, $X$ ray diffraction, Mössbauer spectroscopy 


\section{INTRODUCCIÓN}

Colombia, por la adopción del protocolo de Kyoto (1997) necesita políticas de desarrollo sostenible mediante la optimización de los recursos naturales, la reutilización de desechos, la eficiencia energética y la minimización de todo tipo de contaminantes (Aperador et al., 2009; R. Montoya., 2009; Aperador et al., 2012a; Aperador et al., 2012b; Aperador et al., 2012c). Estas políticas requieren la adopción de tecnologías que permitan el desarrollo de investigaciones relacionadas con la reutilización de algunos desechos industriales y darles un valor agregado. Específicamente, el empleo de dos subproductos industriales (escorias siderúrgicas y cenizas volantes procedentes de la industria del acero y centrales termoeléctricas respectivamente) supondría un gran avance en la innovación de los procesos tecnológicos, mediante el desarrollo de materiales de construcción, alternativos a los cementos Portland tradicionales (Oh et al., 2010; Ravikumar et al., 2010; Diaz et al., 2010; Komljenović et al., 2010; Criado et al., 2010; Criado et al., 2010b). En la fabricación de estos nuevos materiales de construcción no se emiten gases contaminantes, se obtiene un apreciable ahorro energético y se incrementa la vida útil de las estructuras que las componen (Song et al., 2006; Duxson et al., 2007). Las escorias siderúrgicas y las cenizas volantes constituyen dos de los subproductos colombianos con mayor potencialidad para ser empleados como materias primas en la producción de concretos como tecnología alternativa, por un menor consumo de energía para su transformación y preparación, respecto a los concretos Portland tradicionales (Aperador et al., 2009; R. Montoya., 2009; Aperador et al., 2012a).

En este trabajo se utilizaron estos dos subproductos industriales como material cementante incorporado, los cuales se activarían para acelerar la disolución de la estructura y la hidratación de la escoria siderúrgica y de la ceniza volante. En estudios previos se ha demostrado el buen desempeño mecánico a la compresión, mayor densidad estructural y buena durabilidad que ofrecen los concretos alternativos cuando se han mezclado, por separado, con respecto a muestras de concreto Portland tradicional (Oh et al., 2010; Ravikumar et al., 2010; Komljenović et al., 2010; Criado et al., 2010). Las escorias y las cenizas volantes tienen algunos efectos importantes sobre el funcionamiento de estructuras de concreto armado siendo los más importantes: la enorme reducción de la tasa de penetración de los iones cloruro en el concreto, el aumento de la concentración crítica de cloruro respecto a la corrosión inducida y la resistencia al ataque de sulfatos (Rodríguez et al., 2008; Puertas et al., 2009; Duran Atiş et al., 2009; Bernal et al., 2009).

Los objetivos de esta investigación fueron: la evaluación del comportamiento electroquímico del acero estructural embebido en un concreto alternativo elaborado a partir de la mezcla de escoria siderúrgica (GBFS) y de cenizas volantes (C), y la caracterización de los productos de corrosión generados en el proceso. Los concretos fueron expuestos al ataque de agentes agresivos como cloruros.Se emplearon técnicas de caracterización como potencial de circuito abierto (OCP), resistencia a la polarización lineal (LPR) y espectroscopía de impedancia electroquímica (EIS). La interfase acero-concreto fue caracterizada por espectrometría Mössbauer de transmisión a temperatura ambiente y difracción de rayos X (DRX).

\section{PROCEDIMIENTO EXPERIMENTAL.}

Muestras de concreto y preparación de especímenes

Se utilizaron tres clases de cementantes:

a) ceniza volante tipo $\mathrm{F}(\mathrm{C})$ procedente de la central termoeléctrica Termozipa, cuya composición química se registra en la Tabla 1. La pérdida al fuego fue de $4,23 \%$ de la masa total determinada por calcinación de la muestra a $1000{ }^{\circ} \mathrm{C}$, valor asociado principalmente a restos de carbón inquemados. Adicionalmente, se evaluó el porcentaje de sílice reactiva de la ceniza volante siguiendo el procedimiento descrito en lanorma UNE 80-225-93, obteniéndose un valor del $41,23 \%$ en masa.

b) escoria siderúrgica de alto horno (GBFS) con composición química mostrada en la Tabla 1, coeficientes de basicidad $\left(\mathrm{CaO}+\mathrm{MgO} / \mathrm{SiO}_{2}+\mathrm{Al}_{2} \mathrm{O}_{3}\right)$ de $1,0 \mathrm{y}$ calidad $\left(\mathrm{CaO}+\mathrm{MgO}+\mathrm{Al}_{2} \mathrm{O}_{3} / \mathrm{SiO}_{2}+\mathrm{TiO}_{2}\right)$ de 1,73. Como activador alcalino se utilizó una solución de silicato de sodio a una concentración de $5 \%$ de $\mathrm{Na}_{2} \mathrm{O}$ expresado como porcentaje en peso de escoria (ASTM C 989-99).

c) cemento portland tipo I (OPC) de uso común en construcción y composición química relacionada en la Tabla 1.

Los agregados corresponden a grava con tamaño máximo de $17 \mathrm{~mm}$, gravedad específica de 3,12 y absorción de $1,6 \%$; y arena de río con superficie específica de $2380 \mathrm{~kg} / \mathrm{m}^{3}$ y absorción de $3,0 \%$. A partir de los materiales cementantes y agregados se prepararon concretos de $400 \mathrm{~kg} \mathrm{por}^{3}$ de concreto y 0,4 en relación agua/ cementante. 
Tabla 1: Composición química de las cenizas volantes, la escoria siderúrgica de alto horno y el OPC

\begin{tabular}{|c|c|c|c|}
\hline Compuesto & $\begin{array}{c}\text { Cenizas volantes } \\
(\% \text { en masa })\end{array}$ & $\begin{array}{c}\text { Escoria granulada de alto } \\
\text { horno } \\
(\% \text { en masa })\end{array}$ & $\begin{array}{c}\text { OPC } \\
\text { (\% en masa })\end{array}$ \\
\hline $\mathrm{SiO}_{2}$ & 56,6 & 33,7 & 19,05 \\
\hline $\mathrm{Al}_{2} \mathrm{O}_{3}$ & 23,1 & 12,8 & 5,79 \\
\hline $\mathrm{Fe}_{2} \mathrm{O}_{3}$ & 4,6 & 0,48 & 2,94 \\
\hline $\mathrm{CaO}$ & 5,7 & 45,4 & 1,03 \\
\hline $\mathrm{MgO}$ & 0,9 & 1 & \\
\hline $\mathrm{Na}_{2} \mathrm{O}$ & 0,8 & 0,12 & \\
\hline $\mathrm{K}_{2} \mathrm{O}$ & 1,4 & 1,5 & \\
\hline $\mathrm{P}_{2} \mathrm{O}_{5}$ & 0,7 & & \\
\hline $\mathrm{TiO}_{2}$ & 1 & 0,5 & 2,18 \\
\hline $\mathrm{MnO}_{\mathrm{SO}}$ & 0,01 & & 3,29 \\
\hline $\mathrm{SiO}_{2} / \mathrm{Al}_{2} \mathrm{O}_{3}$ & 0,50 & 2,63 & \\
\hline & 2,7 & & \\
\hline
\end{tabular}

Para el estudio se conformaron cuatro tipos de muestras de concreto: Portland (OPC) como referencia, con $100 \%$ de escoria siderúrgica activada alcalinamente (GBFS) y, con escoria siderúrgica activada alcalinamente y adición de ceniza volante (C) del 10\% y 20\% respectivamente. La Tabla 2 muestra el tipo de probeta y su respectiva composición

Tabla 2. Tipo y Conformación de probetas de concreto

\begin{tabular}{|c|c|}
\hline Clase & Composición \\
\hline OPC & $100 \%$ Concreto Portland \\
\hline G100 & $100 \%$ GBFS \\
\hline G90-C10 & $90 \%$ de GBFS $90 \%+10 \%$ ceniza volante \\
\hline G80-C20 & $80 \%$ de GBFS $+20 \%$ de ceniza volante \\
\hline
\end{tabular}

\section{Ensayo electroquímico}

Para la evaluación electroquímica se produjeron cilindros de 76,2 mm de diámetro por 76,2 mm de alto, con una varilla de acero estructural ASTM A 706colocada en el centro de la probeta; el diámetro del acero de refuerzo fue de $6,35 \mathrm{~mm}$. En la figura 1 se muestra el montaje correspondiente, se aprecian detalles como la longitud del acero expuesto $(50 \mathrm{~mm})$, los segmentos de la varilla de refuerzo que fueron pintadas y la ubicación de un epóxico en la interfase. La parte superior de las probetas de concreto se protegió con la sustancia epóxica para evitar entrada preferente de cloruros por la interfase armado/concreto. Los concretos obtenidos de mezclas de escorias siderúrgicas y de cenizas volantes activadas alcalinamente se curaron a una humedad relativa del $90 \%$ durante 28 días previos al desarrollo delos diferentes ensayos.

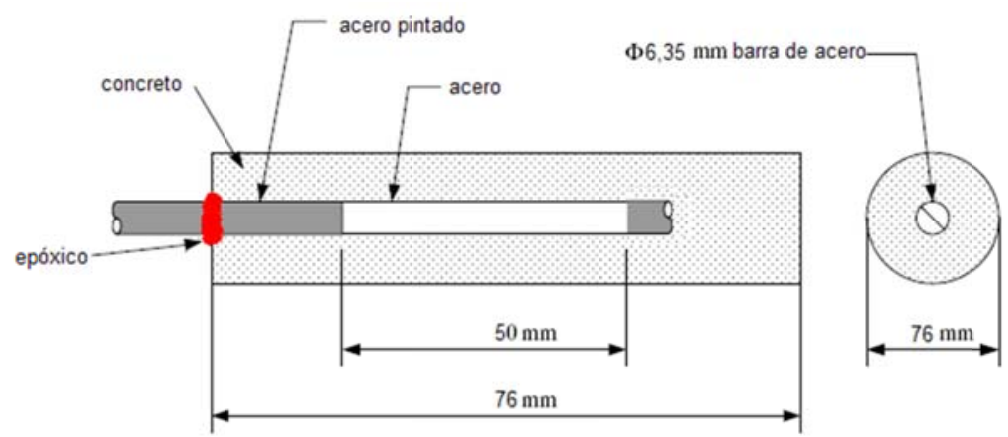

Fig 1: Diagrama esquemático de la muestra de concreto armado.

Para la caracterización electroquímica se empleó un potenciostato/galvanostato Gamry modelo PCl 4 mediante las técnicas de potencial de circuito abierto (ASTM C 876-09), resistencia a la polarización lineal y espectroscopía de impedancia electroquímica. La celda estaba compuesta por un contra-electrodo de grafito, no embebido en el concreto, electrodo de referencia de $\mathrm{Cu} / \mathrm{CuSO}_{4}$ y el acero estructural ASTM A 706 con área de exposición de $10 \mathrm{~cm}^{2}$ como electrodo de trabajo.

En la figura 2 se observa el montaje experimental para el desarrollo de la caracterización electroquímica. Durante el desarrollo de los análisis las diferentes probetas fueron sumergidas en un tanque que contenía agua destilada. Se aplicó un potencial externo $\pm 20 \mathrm{mVy}$ velocidad de $0,125 \mathrm{mV} / \mathrm{s}$ alrededor del potencial de circuito abierto para las pruebas de resistencia a la polarización lineal (LPR). Los diagramas de Nyquist se 
obtuvieron mediante barridos de frecuencia entre $100 \mathrm{kHz}$ y $0,01 \mathrm{~Hz}$ con $10 \mathrm{mV}$ de amplitud en la señal sinusoidal. La medida de potencial de circuito abierto se tomó, hasta su estabilización, a un tiempo de 3,33 horas.

Las medidas electroquímicas para los cuatro tipos de concreto y relacionados en la Tabla 2, se tomaron cada 3 meses hasta los 12 meses. Todas las probetas se sometieron a proceso corrosivo mediante inmersión en una disolución de $\mathrm{NaCl}$ al 3,5\%, extrayéndose solamente por el tiempo necesario para practicar los diferentes análisis.

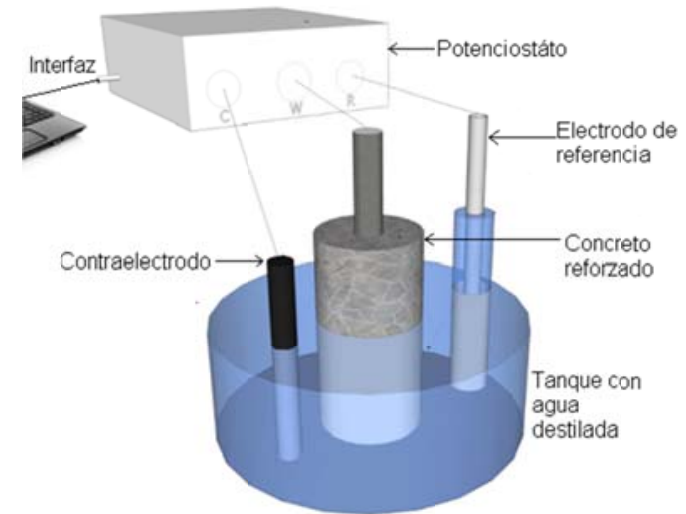

Fig 2: Esquema del montaje experimental para las mediciones electroquímicas.

\section{Ensayos de caracterización}

Para la caracterización del ingreso del ion cloruro se utilizó la norma ASTM C1202.

Para identificar los productos de corrosión generados en el proceso, las muestras de óxidos fueron extraídas de la superficie del acero mediante una lima de diamante. Posteriormente, las muestras de óxidos se llevaron a DRX y Mössbauer para su análisis.

El arreglo experimental en DRX corresponde a goniómetro PW3050/60 ( $\theta / \theta)$,sistema XPERT-PRO con radiación monocromática de $\mathrm{Cu} \mathrm{Ka} 1,54 \AA$, en condiciones de operación a $40 \mathrm{kV}, 40 \mathrm{~mA}$ y $25^{\circ} \mathrm{C}$.El barrido fue desde $2 \theta=20,01^{\circ}$ hasta $2 \theta=99,99^{\circ}$ con un paso de $0,02^{\circ}$ y tiempo de barrido de 1 segundo.Las fases cristalinas se determinaron con la bases datos del equipo. Además, se utilizó el programa MAUD que permite el refinamiento de los difractogramas mediante el método Rietveld. Los resultados encontrados se contrastaron con los reportados por otros investigadores (Bernal et al., 2010).

Los espectros de Mössbauer se estimaron mediante un arreglo típico de espectrometría de ${ }^{57} \mathrm{Fe}$, empleando como fuente una pastilla de ${ }^{57} \mathrm{Co}$ en una matriz de rodio, la cual decae radiactivamente a ${ }^{57} \mathrm{Fe}$ (Rodríguez, RR et al., 2008).

\section{RESULTADOS Y DISCUSIÓN}

Evaluación de la Resistencia a Penetración de Cloruros.

En la figura 3, se muestra la determinación de la permeabilidad al ión cloruro del concreto, midiendo el paso de corriente eléctrica a través del mismo. Las medidas se realizaron a los 28 días de curado. Se observa el comportamiento de los concretos analizados en función de la carga transferida, aquí se obtiene que el concretos obtenidos a partir de las mezclas de subproductos industriales presenta una menor permeabilidad al ion cloruro, en comparación a los OPC.

La medida de la resistividad del hormigón, que se puede extraer a partir del ensayo de penetración a ion cloruro es utilizado para interpretar el valor de la velocidad de corrosión, ya que está intrínsecamente relacionada con el contenido de humedad del hormigón. La prueba rápida de la permeabilidad del ión del cloruro, es esencialmente una medida de la conductividad eléctrica que depende de la estructura del poro y de la química de la solución del mismo. Para un tamaño dado del espécimen y un voltaje aplicado, la corriente inicial registrada se puede tomar como representante de la conductividad eléctrica de la muestra. Se observa que los concretos OPC presentan una conductividad eléctrica más alta que los concretos obtenidos a partir de las mezclas de subproductos industriales. 


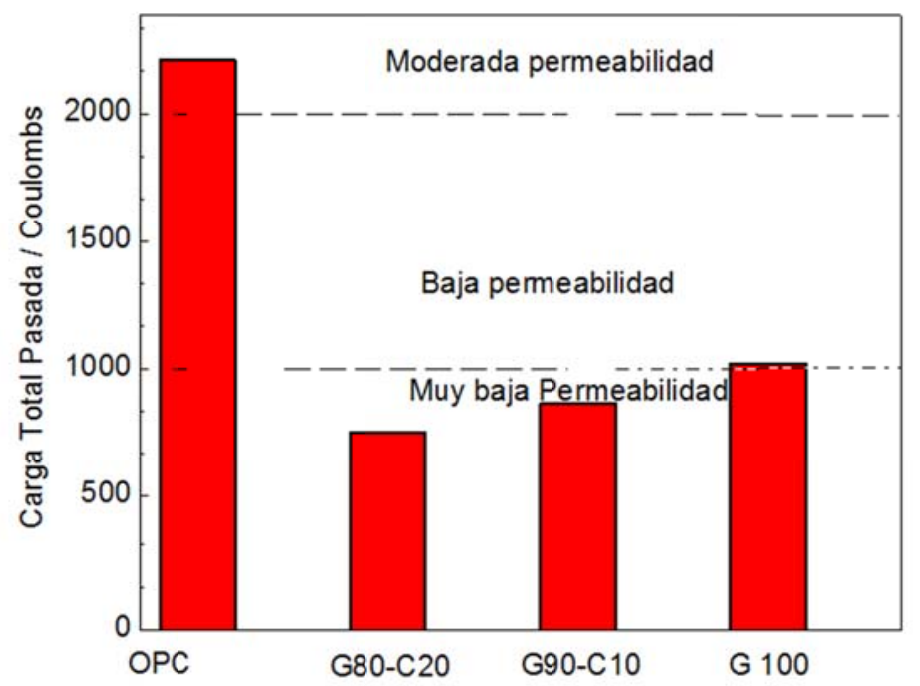

Fig 3. Valores de permeabilidad al ion cloruro de los concretos OPC y alternativos.

\section{Potencial de circuito abierto (Ecorr)}

En la gráfica de la figura 4 se observa el fenómeno corrosivo de los concretos Portland ordinario (OPC), escorias activadas alcalinamente (GBFS) y las mezclas de GPFS $+C$, evaluados termodinámicamente teniendo en cuenta el criterio de la norma ASTM C876-91 para el ensayo y la interpretación. Debido a que el tipo de material en el cual se hallaba el acero embebido es diferente, estos sistemas presentan diversos valores de potencial de reposo (figura 3).

Para los concretos Portland, se advierte una disminución de valores de potenciales de corrosión en función del tiempo. Este tipo de comportamiento es frecuente para la clase de material empleado en el estudio. Se observa un comportamiento de corrosión activa después de los 0 meses de inmersión en la disolución de $\mathrm{NaCl}$ (transcurridos 28 días de curado). A los 3 meses de evaluación (tiempo de inmersión en la disolución) se observa que la tendencia de los potenciales, en los concretos OPC, es decreciente.

Con relación a los concretos $100 \%$ GBFS, la figura 3 muestra un comportamiento en los potenciales similar al encontrado en las muestras de concreto OPC. Sin embargo, el potencial de corrosión a los 0 meses es menor y está relacionado con los diferentes productos de hidratación existentes en cada uno de los concretos.

En concretos cuyas mezclas son GBFS + C con adiciones de cenizas volantes del $10 \%$ y $20 \%$, las medidas de potencial al inicio del estudio ( 0 meses) se ubican en la zona 10\% de probabilidad de corrosión (Figura 3). Los valores de potencial para 3, 6, 9 y 12 meses se sitúan en la zona de pasivación.

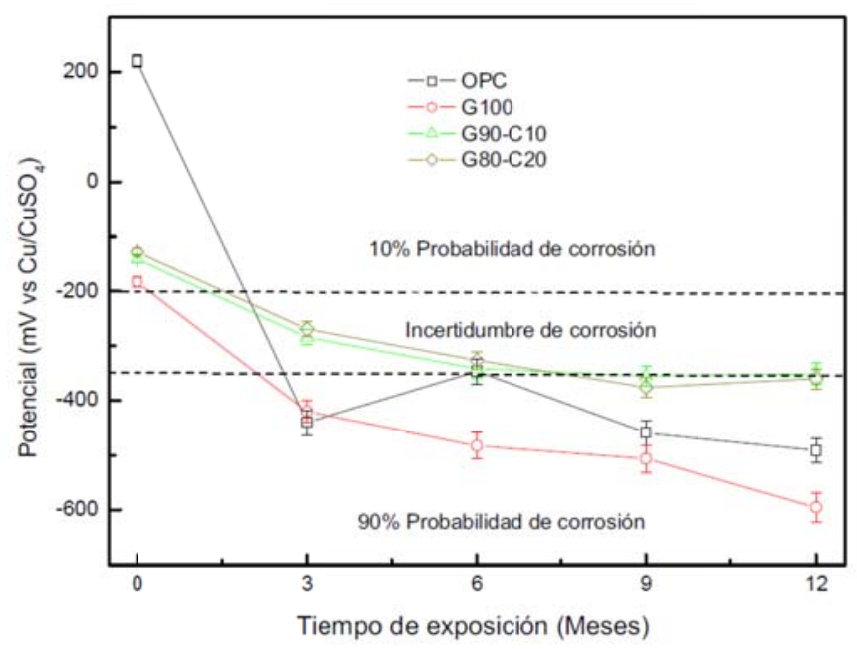

Fig 4:Potencial de corrosión en función del tiempo para los concretos OPC, G100, G90-C10 y G80-C20 con exposición a iones cloruro. 


\section{Resistencia a la polarización lineal (LPR)}

En la figura 5, se presentan los resultados obtenidos en el análisis de LPR para las probetas de concreto reforzado inmersas en la disolución con iones cloruro. Al utilizar el criterio de la resistencia a la corrosión propuesto por C. Andrade et al., 1984, para la predicción del estado del acero en concretos OPC y G100, se pudo observar altos valores en velocidad de corrosión a medida que transcurre el tiempo de inmersión. Analizando los resultados de LPR en concretos G90-C10, se observa estabilización de los valores de resistencia a la polarización a los 6 meses de evaluación. Estas mezclas también indican baja velocidad de corrosión al final del ensayo (12 meses). Una tendencia similar a la reportada anteriormente es mostrada para las mezclas G80-C20con la diferencia que en este último caso, los valores de resistencia a la polarización lineal son mayores.

No obstante, es posible analizar el comportamiento a la corrosión de cada sistema basándose únicamente en el criterio de resistencia a la polarización. Esta puede relacionarse, mediante la ecuación de Ohm, a la encontrada en un circuito eléctrico. De esta forma y según lo observado el potencial de corrosión permanece constante, posterior a los 6 meses de evaluación. Se puede inferir que los concretos OPC y G100 son los que presentan los valores más bajos de resistencia permitiendo el paso de mayores cantidades de corriente. Adicionalmente, se observa que los concretos G90-C10 y G80-C20 son los de mayor resistencia a la polarización posterior a los 6 meses de evaluación. Si se relaciona este hecho con la perdida de especies metálicas, se puede inferir que bajas resistencias a la polarización (Rp) corresponden a altos valores de velocidad de corrosión y viceversa.

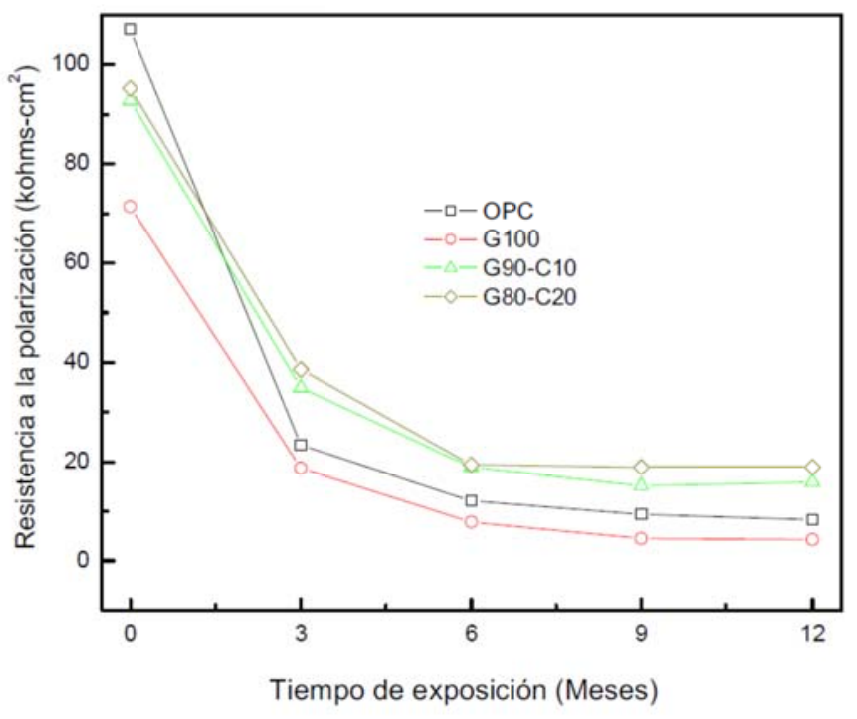

Fig 5:Resistencia a la polarización en función del tiempo para los concretos OPC, G100, G90-C10 y G80C20con exposición a iones cloruro.

\section{Espectroscopía de Impedancia Electroquímica (EIS).}

La técnica de espectroscopía de impedancia que se aplicó a los concretos OPC, G100, G90-C10 y G80C20. Los resultados fueron modelados mediante el circuito descrito en la figura 6 . Este modelo se encuentra reportado en varios trabajos, entre ellos el de Assouli et al., 2005. La resistencia Rc+s, corresponde al dominio de alta frecuencia (entre $100 \mathrm{kHz}$ y el $1 \mathrm{kHz}$ ) y se relaciona con la resistencia eléctrica del concreto, incluyendo la resistencia del electrólito (la resistencia del electrólito es insignificante comparado a la resistencia del concreto). Rc+s puede también incluir las contribuciones de los efectos de la cubierta es decir la interfase entre el electrolito y el concreto. La resistencia R1, corresponde al dominio intermedio de la frecuencia (de $1 \mathrm{kHz}$ a $50 \mathrm{~Hz}$ ), y representa la resistencia en la zona de transición. El elemento de fase constante (CPE1) representa la capacitancia no-ideal en la zona de transición de interfase cemento/agregados cercanos al poro. El dominio de la frecuencia baja $(50 \mathrm{~Hz}$ a $1 \mathrm{mHz})$ se utiliza para evaluar el proceso de la transferencia de carga conjuntamente con el proceso del transporte de masa (Fernández et al, 2004) y corresponde normalmente a la resistencia Rp. CPE2 determina la capacitancia noideal de la superficie de acero. Los valores obtenidos en los cuatro tipos de concretos para cada uno de los parámetros mencionados anteriormente se registran en la Tabla 3. 


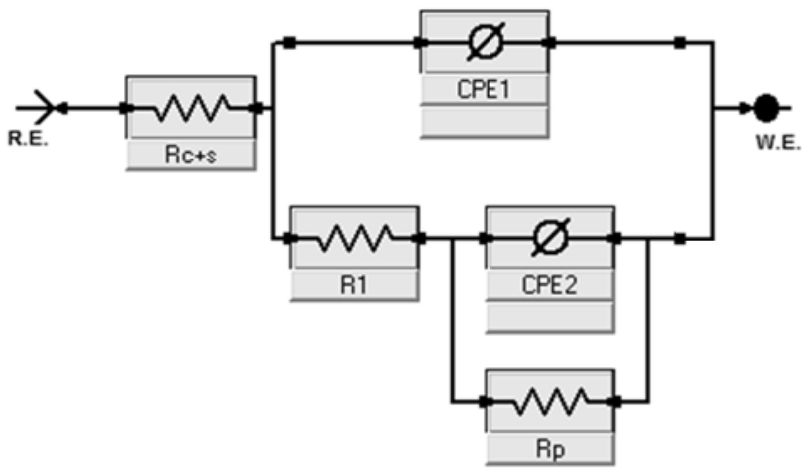

Fig 6: Circuito equivalente correspondiente a los concretos concretos OPC, GBFS, $90 \%$ GBFS $+10 \%$ FA y $80 \% \mathrm{GBFS}+20 \% \mathrm{FA}$ expuestos a ion cloruro evaluados desde 0 hasta 12 meses.

Tabla 3: Parámetros electroquímicos hallados para los concretos OPC, G100, G90-C10 y G80-C20 expuestos a iones cloruro.

\begin{tabular}{|c|c|c|c|c|c|c|c|}
\hline $\begin{array}{c}\text { Nivel de } \\
\text { evaluación }\end{array}$ & $\begin{array}{r}R_{\mathrm{c}+\mathrm{s}} \\
\Omega \mathrm{cm}^{2}\end{array}$ & $\begin{array}{c}\text { CPE1 } \\
\mathrm{F} \mathrm{cm}^{-2} \mathrm{~s}^{-\left(1-\alpha_{1}\right)}\end{array}$ & $\alpha_{1}$ & $\begin{array}{c}\mathrm{R}_{1} \\
\mathrm{k} \Omega \mathrm{cm}^{2}\end{array}$ & $\begin{array}{c}\mathrm{CPE2} \\
\mathrm{F} \mathrm{cm}^{-2} \mathrm{~s}^{-\left(1-\alpha_{2}\right)}\end{array}$ & $a_{2}$ & $\begin{array}{c}R_{\mathrm{p}} \\
\mathrm{k} \Omega \mathrm{cm}^{2}\end{array}$ \\
\hline \multicolumn{8}{|c|}{ G80-C20 } \\
\hline 0 & 60,2 & $35 \times 10^{-6}$ & 0,59 & 29,2 & 0,13 & 0,52 & 59,13 \\
\hline 1 & 68,0 & $355 \times 10^{-6}$ & 0,75 & 8,67 & $90 \times 10^{-3}$ & 0,85 & 35,6 \\
\hline 2 & 53,2 & $290 \times 10^{-6}$ & 0,87 & 3,45 & $54 \times 10^{-3}$ & 0,93 & 23,31 \\
\hline 3 & 40,6 & $70 \times 10^{-6}$ & 0,93 & 2,72 & $34 \times 10^{-3}$ & 0,86 & 22,19 \\
\hline 4 & 27,3 & $21 \times 10^{-6}$ & 0,86 & 2,57 & $24 \times 10^{-3}$ & 0,72 & 20,75 \\
\hline \multicolumn{8}{|c|}{ G90-C10 } \\
\hline 0 & 59,8 & $24 \times 10^{-6}$ & 0,65 & 24,34 & 0,54 & 0,72 & 56,36 \\
\hline 1 & 72 & $295 \times 10^{-6}$ & 0,84 & 7,38 & $82 \times 10^{-3}$ & 0,69 & 32,26 \\
\hline 2 & 49,3 & $221 \times 10^{-6}$ & 0,69 & 4,87 & $49 \times 10^{-3}$ & 0.87 & 21,54 \\
\hline 3 & 43,2 & $49 \times 10^{-6}$ & 0,75 & 3,21 & $31 \times 10^{-3}$ & 0,82 & 20,33 \\
\hline 4 & 42,9 & $13 \times 10^{-6}$ & 0,89 & 2,02 & $20 \times 10^{-3}$ & 0,79 & 18,32 \\
\hline \multicolumn{8}{|c|}{ G100 } \\
\hline 0 & 63,27 & $294 \times 10^{-6}$ & 0,83 & 10,42 & $115 \times 10^{-3}$ & 0,87 & 43,32 \\
\hline 1 & 43,93 & $169 \times 10^{-6}$ & 0,91 & 8,64 & $76 \times 10^{-3}$ & 0,69 & 23,95 \\
\hline 2 & 40,21 & $119 \times 10^{-6}$ & 0,82 & 5,45 & $89 \times 10^{-3}$ & 0,74 & 14.32 \\
\hline 3 & 39,8 & $75 \times 10^{-6}$ & 0,78 & 3,72 & $81 \times 10^{-3}$ & 0,78 & 8,33 \\
\hline 4 & 38,52 & $45 \times 10^{-6}$ & 0,92 & 1,02 & $51 \times 10^{-3}$ & 0,87 & 5,32 \\
\hline \multicolumn{8}{|c|}{ OPC } \\
\hline 0 & 64,3 & $117 \times 10^{-6}$ & 0,81 & 15.9 & 0.703 & 0,86 & 158 \\
\hline 1 & 36,7 & $38 \times 10^{-6}$ & 0,87 & 1.13 & $589 \times 10^{-3}$ & 0,75 & 14,4 \\
\hline 2 & 42,7 & $20 \times 10^{-6}$ & 0,79 & 0.80 & $458 \times 10^{-3}$ & 0,78 & 11,4 \\
\hline 3 & 34,1 & $24 \times 10^{-6}$ & 0,84 & 0.73 & $433 \times 10^{-3}$ & 0,83 & 10,3 \\
\hline 4 & 29,1 & $14 \times 10^{-6}$ & 0,92 & 1.593 & $257 \times 10^{-3}$ & 0,92 & 8,4 \\
\hline
\end{tabular}

Los diagramas Nyquist de las figuras 7 y 8 corresponden a los concretos G90-C10, G80-C20, G100 y OPC a los 0 días de evaluación, es decir, a los 28 días de curado (figura 6) y a los 12 meses de inmersión en una disolución de cloruros. Al comparar las figuras 7 y 8 se obtiene una disminución de todos los parámetros electroquímicos hallados, luego del sometimiento a los 12 meses a iones cloruro, los valores de los parámetros que más han disminuido son la resistencia a la solución y la resistencia a la polarización. En el primer nivel evaluado (3 meses) se encuentra una disminución elevada debido al ingreso del ion cloruro en los diferentes concretos, se observa el mismo comportamiento para los demás niveles estudiados en los que se visualiza una disminución por el desplazamiento hacia la izquierda de cada una de las diagramas Nyquist. El modelamiento del sistema corresponde al mostrado en la figura 6. Este modelo explica, electroquímicamente, el efecto de iones cloruro sobre la ruptura de la pasividad del acero, y es el que mejor se ajusta en este estudio. En el circuito de la figura 6, la primera resistencia se opone a los iones de la 
solución al entrar en el concreto este valor es pequeño para los primeros niveles ya que la cantidad de iones es mínimo, por lo tanto entran con facilidad, luego en los siguientes niveles estos concretos están saturados de solución agresiva por lo tanto este valor aumenta.

La resistencia (R1) representa la interfase de concreto/agregados, el valor de esta resistencia varía dependiendo del nivel evaluado (tabla 2). En los primeros niveles se genera mayor resistencia de los iones cuando pasan a través de los agregados, esta resistencia empieza a disminuir ya que la resistividad del concreto empieza a decaer cuando ingresan iones cloruro al sistema. En esta misma interfase se halla en paralelo a la R1 el elemento de fase constante CPE1 y representa las imperfecciones superficiales de la interfase. Los valores de CPE en cada uno de los casos es alto, por lo tanto los iones toman otra trayectoria representada por la resistencia hallada entre el concreto y la capa pasivante del acero. El valor de la resistencia a la polarización (R2) es cada vez menor ya que la oposición en los primeros niveles es mayor debido a la capa de pasivación presente en el sistema. Esta capa empieza a verse comprometida luego de que cierta cantidad de iones cloruro han entrado hasta la interfase, es decir a medida que aumenta el porcentaje de iones cloruro en esta interfase. Por lo tanto, el valor de la resistencia a la polarización es cada vez menor lo que indica un aumento en la velocidad de corrosión para los concretos OPC y G100 y una disminución de la velocidad de corrosión para los concretos G90-C10, G80-C20, ya que el comportamiento es inverso al presentado por el concreto OPC. El elemento de fase constante correspondiente a esta interfase y conectado en paralelo a la resistencia a la polarización muestra una disminución de este parámetro, fenómeno generado porque en cada nivel este elemento ofrece un mayor paso de los iones generando el deterioro superficial del acero, como lo reportan Holden (1983) y Popovics (1983).

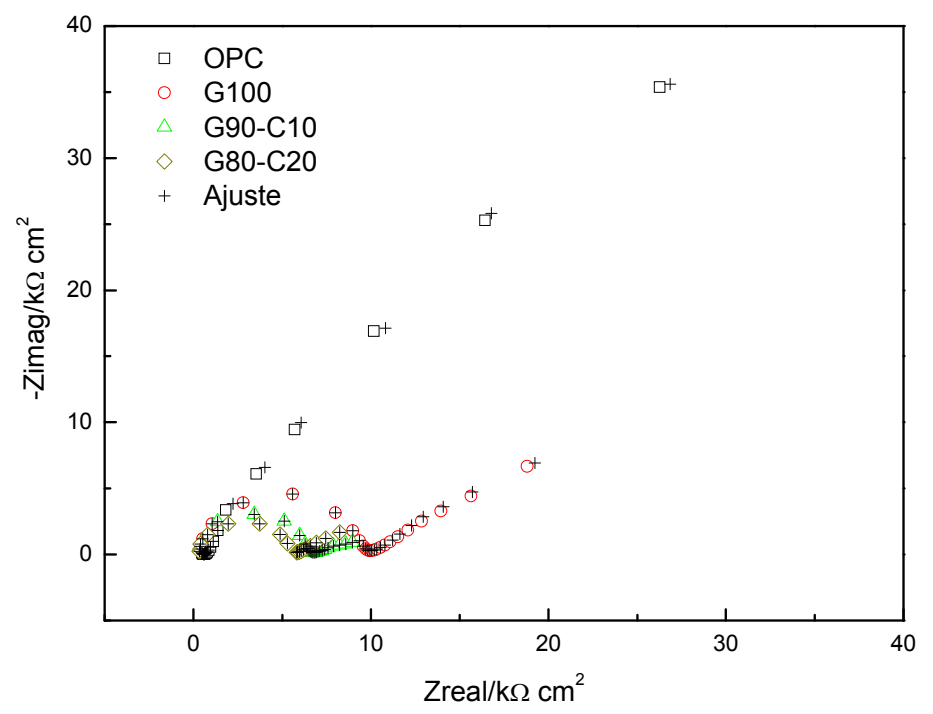

Fig. 7: Diagrama de Nyquist correspondiente a los concretos concretos OPC, OPC, G100, G90-C10 y G80C20 expuestos a iones cloruro evaluados a los 28 días posteriores al curado.

\section{Caracterización productos de corrosión}

Los análisis con DRX de las superficies del acero de refuerzo permitieron evidenciar la presencia de óxidos e hidróxidos de hierro para todas las muestras estudiadas (OPC, OPC, G100, G90-C10 y G80-C20). Esta evaluación fue realizada para las muestras de concretos después de 12 meses de inmersión.

Otros compuestos que aparecen en el análisis de DRX, se atribuyen a los componentes propios de los agregados. En la figura 9, parte superior, se observa el espectro DRX correspondiente al concreto GBFS expuesto al ion cloruro. En este difractograma están presentes las siguientes fases: C: calcita, G: goethita, AN: Andradita, MG: magnetita, W: Wustita, L: lepidocrocite, HM: hematite y Q: cuarzo. La fase Q presenta alta intensidad, se atribuye a la matriz de concreto (material cementicio y agregados). Los óxidos encontrados sobre la superficie del acero son MG y W, y se presentan con mayor intensidad respecto a los demás excluyendo al cuarzo. Los hidróxidos hallados pertenecen a G, L, y HM. Tanto los óxidos como los hidróxidos registrados en estos espectros se presentan en la mayoría de los procesos de corrosión independientemente de si los concretos son OPC o alternativos. 


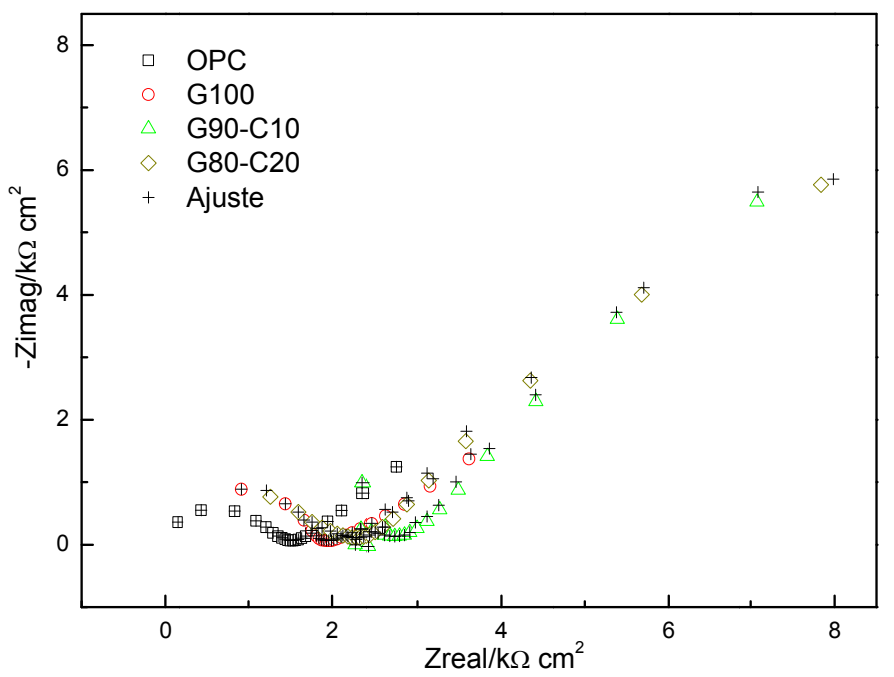

Fig 8. Diagrama de Nyquist correspondiente a los concretos concretos OPC,, G100, G90-C10 y G80-C20 expuestos a iones cloruro evaluados en los 12 meses de exposición.

El espectro correspondiente a los concretos OPC y G90-C10 y G80-C20, también incluido en la figura 8, presentan las mismas fases pero con picos de menor intensidad.

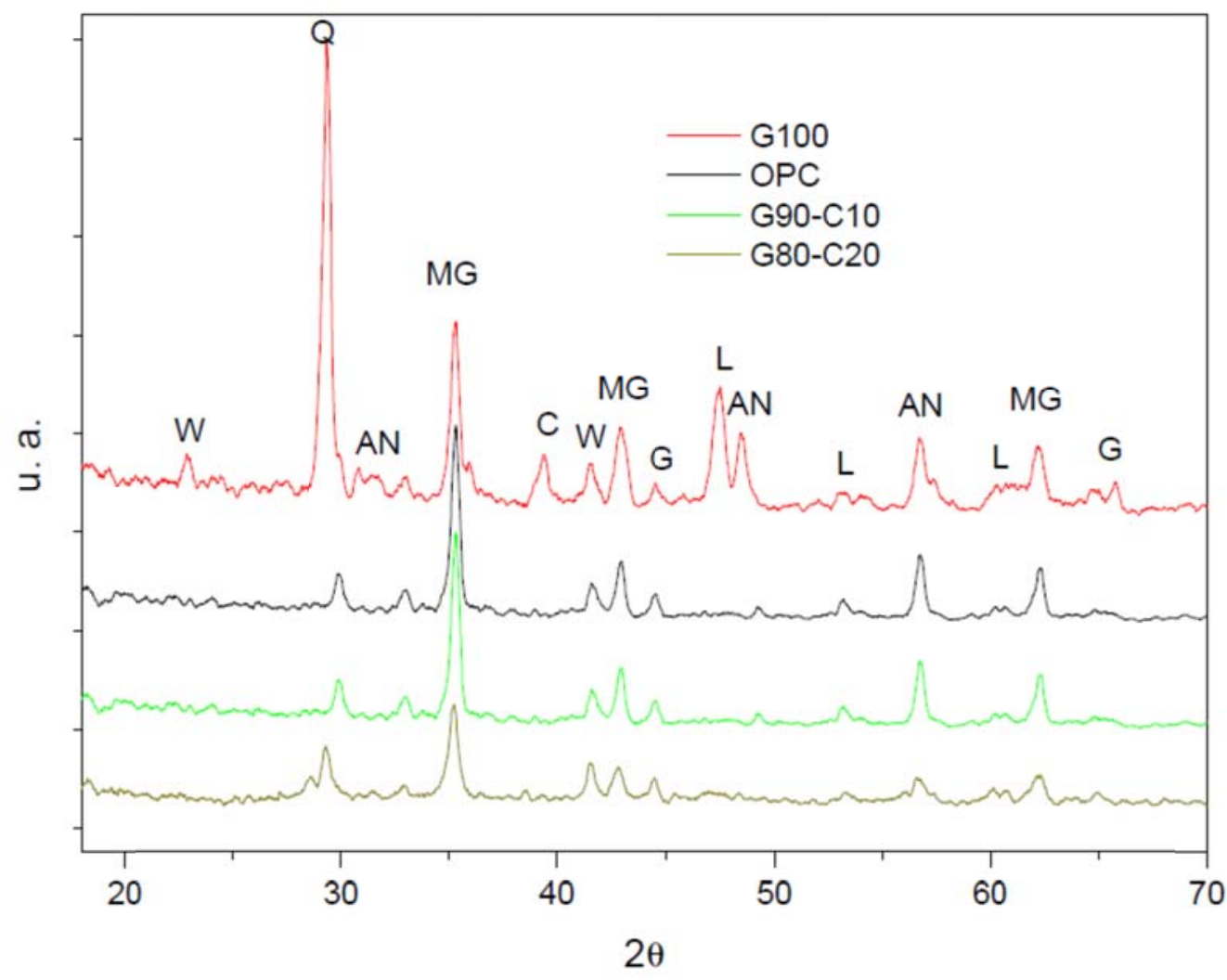

Fig 9: Espectros de DRX para los concretos OPC, G100, G90-C10 y G80-C20 expuestos a iones cloruro.

En la Figura 10 se muestran los espectros Mössbauer tomados a temperatura ambiente, a) OPC, b) G100, c) G90-C10 y d) G80-C20. En las muestras OPC, G100, G90-C10y G80-C20expuestos a ion cloruro y para obtener el mejor ajuste del espectro Mössbauer se utilizaron tres sextetos y dos dobletes para todos los concretos. Dos de los sextetos, ajustados con campos magnéticos hiperfinos (Bf) 47,9 y 47,3 T, desdoblamientos cuadrupolares $(\Delta)$ cero para ambas, y desvíos isoméricos $(\delta)$ entre 0,14 y $0,63 \mathrm{~mm} / \mathrm{s}$, respectivamente, se atribuyen a una fase espinela de magnetita $\left(\mathrm{Fe}_{3-\mathrm{x}} \mathrm{O}_{4}\right)$ o posible combinación de $\mathrm{Fe}_{3-\mathrm{x}} \mathrm{O}_{4} /$ maghemita $\left(\mathrm{\gamma}-\mathrm{Fe}_{2} \mathrm{O}_{3}\right)$. El otro sexteto se ajustó con parámetros entre, $\mathrm{Bf}=29,8 \mathrm{~T}, \Delta=-0,023 \mathrm{~mm} / \mathrm{s}$ y $\delta=$ 
$0,05 \mathrm{~mm} / \mathrm{s}$, se atribuye a una fase de Fe. Para uno de los dobletes se encontró los valores entre $\Delta=1,23$ $\mathrm{mm} / \mathrm{s}$ y $\delta=0,04 \mathrm{~mm} / \mathrm{s}$, correspondiente a la presencia de $\mathrm{Fe}^{2+} \mathrm{O}$; en cuanto al otro doblete se encontró el rango de $\Delta=0,69 \mathrm{~mm} / \mathrm{s}$ y $\delta=0,86 \mathrm{~mm} / \mathrm{s}$, correspondiente a la presencia de $\mathrm{Fe}{ }^{3+} \mathrm{O}(\mathrm{OH})$.

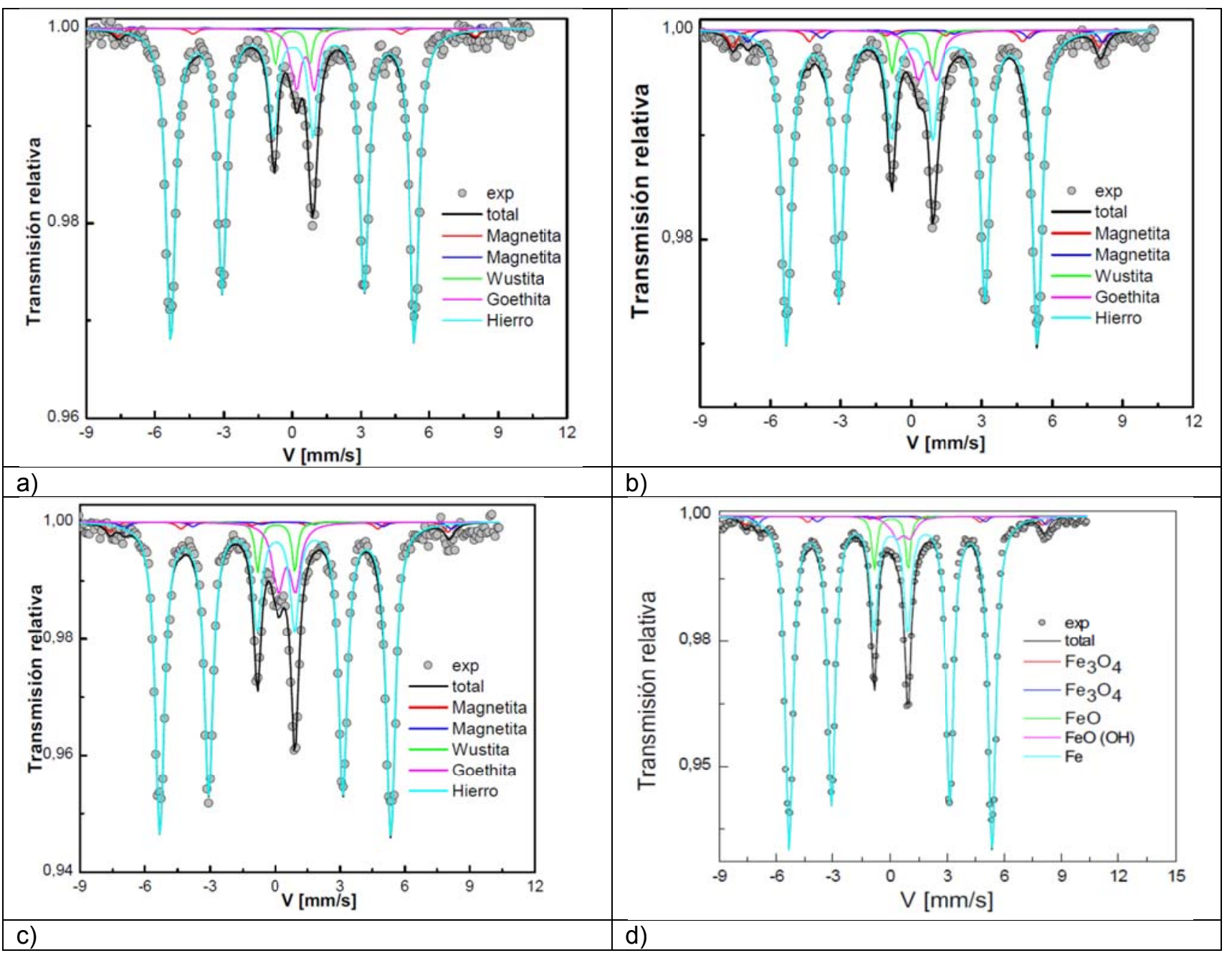

Fig 10: Espectro Mössbauer para los concretos a) OPC, b)G100, c) G90-C10 y d) G80-C20 expuestos a iones cloruro.

En la tabla 4, se reportan los resultados porcentuales de óxidos e hidróxidos presentes en cada uno de los concretos. Para todos los casos se observa un alto porcentaje de hierro. En los concretos G90-C10y G80C20 la proporción de hidróxido goethita presente es superior comparado con los otros productos hallados (Magnetita y Wustita), esto indica que la mayoría de los productos encontrados son goethita. Los porcentajes correspondientes a los productos de corrosión sobre el acero embebido en G90-C10y G80-C20 son aproximadamente similares entre sí, tan solo el contenido de la fase goethita representa el mayor porcentaje de escoria alcalina. En cuanto a la comparación de los concretos activados en comparación con el concreto de referencia (OPC), los productos de corrosión disminuyen en un promedio del $6 \%$.

Tabla 4: Relación de fases por espectro Mössbauer de los productos hallados para concretos OPC, G100, G90-C10y G80-C20 expuestos a iones cloruro.

\begin{tabular}{|c|c|c|c|c|}
\hline Fase & OPC & GBFS & $\begin{array}{c}90 \% \text { GBFS } \\
+10 \% \text { FA }\end{array}$ & $\begin{array}{c}80 \% \text { GBFS } \\
+20 \% \text { FA }\end{array}$ \\
\hline Magnetita (\%) & $6.76 \pm 0.0011$ & $4.96 \pm 0.0016$ & $3,4 \pm 0.0011$ & $3,2 \pm 0.0014$ \\
\hline Wuestita (\%) & $3.31 \pm 0.0023$ & $5,85 \pm 0.0012$ & $4,05 \pm 0.0021$ & $4,2 \pm 0.0026$ \\
\hline Goethita (\%) & $6.63 \pm 0.0043$ & $9,41 \pm 0.0028$ & $8,28 \pm 0.0029$ & $8,5 \pm 0.0032$ \\
\hline Hierro (\%) & $83.30 \pm 0.0018$ & $79,78 \pm 0.0019$ & $84,27 \pm 0.0010$ & $84,1 \pm 0.0021$ \\
\hline
\end{tabular}

La Wustita sólo es estable a altas temperaturas, por lo tanto a temperatura ambiente se presenta en forma de residuo de forma laminar ofreciendo muy poca protección a largo plazo para el acero en exposición ambiental o ambiente rico en cloruros. La goethita formada tiene alto porcentaje debido a la disolución y 
precipitación de lepidocrocita la cual se transforma en goethita, debido a esto no aparece lepidocrocita en el espectro Mössbauer (Aperador et al., 2012c).

La información arrojada por estas técnicas DRX y Mössbauer es análoga debido a que sobre la superficie del acero de refuerzo embebido en los diferentes concretos OPC se encontró la presencia de los óxidos e hidróxidos de hierro en las muestras analizadas. Además, se estableció la formación de la magnetita $\left(\mathrm{Fe}_{3} \mathrm{O}_{4}\right)$, Wustita $\left(\mathrm{Fe}^{2+} \mathrm{O}\right)$ y goethita $\left(\mathrm{Fe}^{3+} \mathrm{O}(\mathrm{OH})\right)$ como principales productos de corrosión. Otros productos como lepidocrocita y Hematita son hallados con menor intensidad, esto posiblemente se debe al bajo porcentaje de hidróxidos presentes en las muestras analizadas, también debido a la mezcla con el hierro debido a que poseen parámetros similares.

\section{CONCLUSIONES}

Los concretos obtenidos de mezclas de escorias siderúrgicas y de cenizas volantes activadas alcalinamente sometidos al ataque de ion cloruro exhiben un comportamiento similar a los concretos OPC y GBFS, las diferencias se asocian a los potencial de corrosión que se estabiliza luego de los 6 meses de evaluación. Esto indica que el acero embebido en el concreto de mezclas pasa de un estado de corrosión inactiva a uno de un $50 \%$ de probabilidad de corrosión. La resistencia a la polarización corrobora los resultados obtenidos por medidas de Ecorr, en donde los concretos GBFS +FA muestran una disminución de la resistencia a la polarización en los dos primeros niveles. Después del tercer nivel aumenta el valor de la resistencia indicando remoción de la capa de pasivación. Los concretos OPC y GBFS, sometidos a las mismas condiciones, muestran una disminución en cada uno de los niveles estudiados lo que indica que el deterioro de la barra de acero sigue su proceso de degradación.

La técnica de espectroscopía de impedancia electroquímica mostró ser una herramienta apropiada para obtener en detalle la información sobre las características de los diferentes concretos en especial en la interfase presente en este material. Además, se pueden cuantificar los cambios presentados en cada una de las interfases a través de la equivalencia del circuito eléctrico.

Los concretos OPC, GBFS, $90 \%$ GBFS $+10 \% \mathrm{FA}$ y $80 \%$ GBFS $+20 \% \mathrm{FA}$ expuestos a ion cloruro muestran la presencia de los óxidos e hidróxidos de hierro en todas las muestras analizadas. Se encontraron los mismos productos de corrosión (magnetita $\left(\mathrm{Fe}_{3} \mathrm{O}_{4}\right)$, Wustita $\left(\mathrm{Fe}_{2+} \mathrm{O}\right)$ y goethita $\left(\mathrm{Fe}_{3+} \mathrm{O}(\mathrm{OH})\right.$ ). Los concretos OPC y GBFS mostraron un aumento de los productos de corrosión comparado con las mezclas de GBFS +FA.

\section{AGRADECIMIENTOS}

Los autores del presente trabajo expresan su agradecimiento a la dirección de investigaciones de la Escuela Colombiana de Ingeniería "Julio Garavito" y la Universidad Militar Nueva Granada por el financiamiento recibido para su ejecución.

\section{REFERENCIAS}

Brune, D., y Hultquist G. Corrosion of a stainless steel with low nickel content under static conditions, Biomaterials, 6, 265-268 (1985).

De Mello, J.D.B. y De S. Balsamo, P.S., Comportamiento Tribológico de Aceros Inoxidables para Cubertería, Información Tecnológica, 17 (6), 57-62 (2006).

Andrade C., Castelo V., Alonso C., González J. Determination of the corrosion rate of steel embedded in concrete by the polarization resistance and ac impedance methods, ASTM-STP 906, ASTM, Philadelphia, PA, 43-63 (1984).

Aperador W., Mejía de Gutiérrez R., Bastidas D.M, Corrosion resistance of carbonated alkali-activated slag concrete, Corros Sci, 51(4), 2027-2033 (2009).

Fernández J.L, González E.L., Brown S.A. y Batic O.R. Evolución de la porosidad de pastas de cemento portland por la incorporación de una puzolana natural. Información Tecnológica, 15(5), 37-40 (2004)

Aperador W., Bastidas D., Bautista J, Mechanical properties and absorption of chlorides in alkali activated slag concrete and exposed to carbonation. Rev. Fac. Ing. Univ. Antioquia, 62 (1) 67-73 (2012a).

Aperador W., Bautista Ruíz J. H., de Jesús Torres R, Corrosion of Reinforcing Bars Embedded in Alkaliactivated Slag Concrete Subjected to Chloride Attack. Materials Research, 15 (1) 57-62 (2012b). 
Aperador W., Vera E., Mejía De Gutiérrez R. Corrosion behavior of steel bar embedded in alkali activated slag concrete subjected to carbonation and chloride attack. Dyna, 171 (1) 80-87 (2012c).

Assouli, B.F., Simescu, G. Debicki e Idrissi H. Detection and Identification of concrete cracking during corrosion of reinforcing concrete by acoustic emission coupled to the electrochemical techniques. NDT\&E International, 38 (2), 682-689(2005).

ASTM A706 / A706M - 09b Standard Specification for Low-Alloy Steel Deformed and Plain Bars for Concrete Reinforcement, West Conshohocken, PA, American Society for Testing and Materials, 2009.

ASTM C 876-99 Standard, Standard test for half-cell potentials of uncoated reinforcing steel in concrete, West Conshohocken, PA, American Society for Testing and Materials, 1999.

ASTM C 989-99 Standard, Standard specification for ground granulated blast-furnace slag for use in concrete and mortars, West Conshohocken, PA, American Society for Testing and Materials, 1999.

Bernal S., De Gutierrez R., Delvasto S., Rodriguez E. Performance of an alkali-activated slag concrete reinforced with steel fibers. Constr. Build. Mater.24 (2)208-214(2010).

Criado M., Fernández Jiménez A., Palomo A. Effect of sodium sulfate on the alkali activation of fly ash. Cement Concrete Comp. 32 (8) 589-594(2010a).

Criado M., Fernández-Jiménez A., Palomo A. Alkali activation of fly ash. Part III: Effect of curing conditions on reaction and its graphical description. Fuel. 89 (11) 3185-3192(2010b).

Diaz E.I., Allouche E.N., Eklund S. Factors affecting the suitability of fly ash as source material for geopolymers. Fuel. 89 (5) 992-996(2010).

Duran Atiş C., Bilim C., Çelik Ö., Karahan O. Influence of activator on the strength and drying shrinkage of alkali-activated slag mortar. Constr. Build. Mater.23 (1) 548-555(2009).

Duxson P., Fernández-Jiménez A., Provis J.L., Lukey G.C., Palomo A., Deventer J.S.J. van Geopolymer technology: The current state of the art. J. Mater. Sci. 42 (21) 2917-2933 (2007).

Komljenović M., Baščarević Z., Bradić V. Mechanical and microstructural properties of alkali-activated fly ash geopolymersJ. Hazard. Mater. 181 (1) 35-42(2010).

Montoya R., Aperador W., Bastidas D.M. Influence of conductivity on cathodic protection of reinforced alkaliactivated slag mortar using the finite element method. Corrosion Sci.51 (5) 2857-2862(2009).

Oh J.E., Monteiro P.J.M., Jun S.S., Choi S., Clark S.M. The evolution of strength and crystalline phases for alkali-activated ground blast furnace slag and fly ash-based geopolymers. Cement Concrete Res. 40 (1)189196(2010).

Puertas F., Palacios M., Gil-Maroto A., Vázquez T. Alkali-aggregate behavior of alkali-activated slag mortars: Effect of aggregate type. Cement Concrete Comp. 31 (5) 277-284(2009).

Ravikumar D., Peethamparan S., Neithalath N. Structure and strength of $\mathrm{NaOH}$ activated concretes containing fly ash or GGBFS as the sole binder. Cement Concrete Comp. 32 (6)399-410(2010).

Rodríguez E., Bernal S., Mejía de Gutiérrez R., Puertas F. Alternative concrete based on alkali-activated slag. Mater. Constr. 58 (1) 53-67 (2008).

Rodríguez R.R., Pérez-Alcáraz G.A., Sánchez H., Greneche J.M. Milling time effects on the magnetic and structural properties of the $\mathrm{Fe}_{70} \mathrm{Si}_{30}$ system. Microelectron J 39 (11) 1311-1313 (2008).

Song H.-W., Saraswathy V. Studies on the corrosion resistance of reinforced steel in concrete with ground granulated blast-furnace slag-An overview. J. Hazard. Mater. 138 (2) 226-233(2006).

UNE 80-225-93 Standard: Test Methods for Cements: Chemical Analysis. Determination of the reactive silica (SiO2) content in cements, pozzolans and fly ashes.- AENOR, Calle Génova n 6, 28004-MADRID-Spain. 